SESSION II. ATMOSPHERES - Chair: André Maeder 


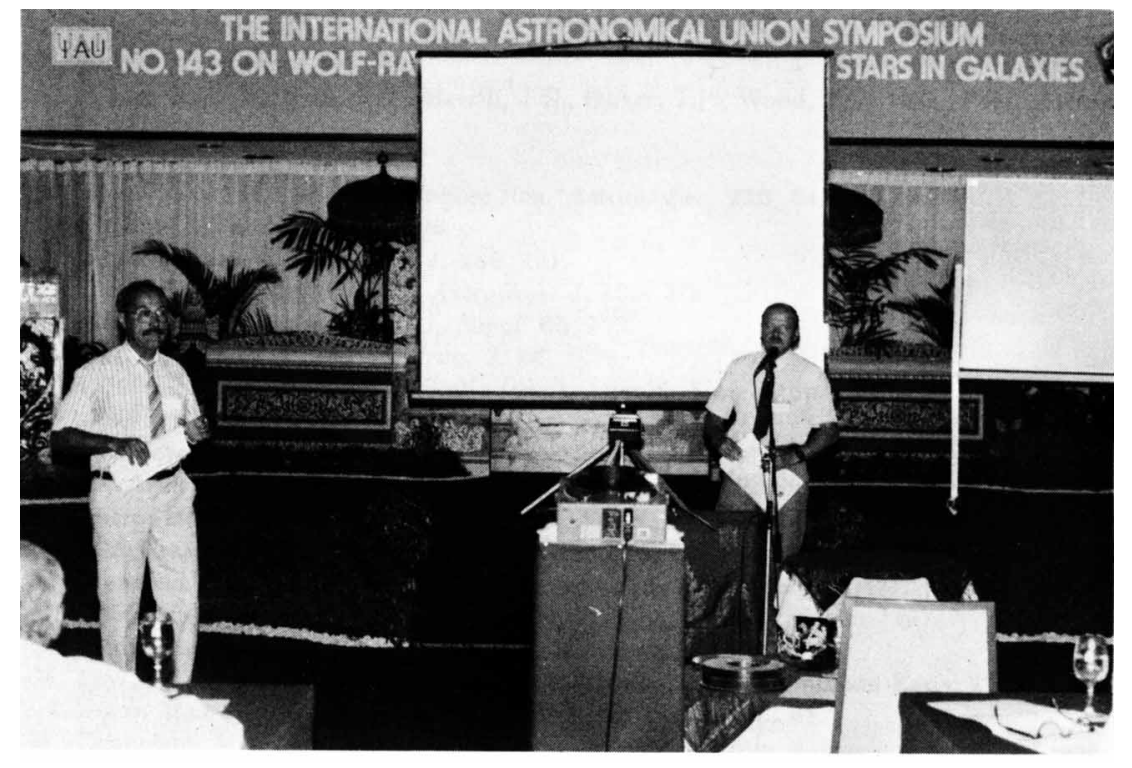

André Maeder chairing, Tiit Nugis 


\title{
OBSERVATIONS VERSUS ATMOSPHERIC MODELS OF WR STARS
}

\author{
WERNER SCHMUTZ \\ Joint Institute for Laboratory Astrophysics \\ University of Colorado and National Institute of Standards and Technology \\ Boulder, CO 80309-0440, USA
}

\begin{abstract}
Current knowledge of Wolf-Rayet continua is reviewed and observed continuum energy distributions are compared with model predictions. Good agreement is found. For WN stars a large range in intrinsic color is found for each subclass and the so far unexplained large range in the observed reddening-free continuum indices can be understood on the basis of atmospheric models. The strength of the continuum discontinuities determines the value of the index. Because of severe blending by the emission lines in WC spectra it can not be decided whether the intrinsic colors of WC stars of a given spectral type vary over a range of values or whether all stars of a given spectral type are similar. The reliability of model calculations for Wolf-Rayet stars is further tested by comparing observed and predicted correlations of helium line strengths. Satisfactory agreement is found for the Kiel/JILA models but not for the Bhatia-Underhill models. Methods used to determine the reddening due to interstellar extinction are reviewed and their accuracies estimated.
\end{abstract}

\section{Introduction}

This paper aims to compare observations of Wolf-Rayet spectra with theoretical predictions. The observations are taken from the literature and since most of the recently published papers that are of relevance to Wolf-Rayet atmospheres are cited here, this paper is also an observational review. The results of atmospheric calculations are taken mostly from the model grids published by Schmutz, Hamann, and Wessolowski (1989) and Schmutz et al. (1990) (Kiel models); but atmospheric models calculated with the code of Hillier (1987a) (JILA models) could have been used as well since both codes-although developed independently-yield the same results. The theory of Wolf-Rayet atmospheres is reviewed by Hillier (this symposium) who discusses model assumptions and calculation techniques and the reader is referred to his paper for more information about the model atmospheres. Here, results from models with atmospheres that consist only of pure helium are used. We may term these models "first generation" or "standard" models, in contrast to the more elaborate calculations that now also include metals (see e.g. Hamann or Hillier, this symposium).

Theory and observations can be compared in many different ways. Here we focus on three aspects: 1) What is known about the continua of Wolf-Rayet stars; 2) How the predicted model energy distributions compare to the observations; and 3) How the predicted helium line strengths compare with those observed. These comparisons allow one to judge the reliability of the model calculations. 


\section{Non-Variability}

Every Wolf-Rayet star monitored with high precision has shown some variability. Before starting with comparisons between model calculations and observations it is useful to think about what tl:e observed variabilities in line profiles and continuum fluxes imply for an analysis. The crucial question is how large are these variations. For most single Wolf-Rayet stars the observed line profile variabilities are only minor perturbations on a well defined mean profile. This is true for lines in the UV (see e.g., St-Louis et al. 1989) as well as in the optical (e.g. McCandliss 1988). The deviations from the mean profile are generally not larger than the deviations of the synthetic profiles from the observed (mean) profile. Thus, it is obvious that model atmospheres are not complex enough to reproduce such profile variations and that the inferred stellar parameters are not affected by the line profile variations.

Short time-scale (hours) photometric variations of single Wolf-Rayet stars are usually below $0.01 \mathrm{mag}$ (e.g. Monderen et al. 1988). Some stars have larger amplitudes, (e.g. HD 104994, van Genderen, van der Hucht, and Larsen 1990), but even in these cases the amplitudes are only of the order of $0.02 \mathrm{mag}$ or less. To recalibrate old photometric measurements, Schmutz and Vacca (1990) compared the photometric fluxes of northern Wolf-Rayet stars determined by Westerlund (1966), with those measured by Massey (1984). They found that the differences in the $b-v$ colors of most $(73 \%)$ of the Wolf-Rayet stars have a standard deviation of only $0.012 \mathrm{mag}$ and that the differences in the $v$ magnitudes have $\sigma=0.038$ mag. This agreement over a time-span of about 18 years implies that: (1) the two authors observed very carefully (in fact the agreement is even slightly better than the authors claimed their data would be) and (2) most Wolf-Rayet stars are stable within $\approx 0.02 \mathrm{mag}$ not only over short time-scales but also over longer time-scales (20 years).

It is a common belief that the observed variations should be interpreted as (small?) deviations from a homogeneous spherically symmetric atmosphere rather than reflecting variations of the stellar parameters. But even if the variations reflect changes of the stellar parameters they are so small that they can be ignored. If we assume that the bolometric luminosity remains constant, an amplitude of $0.03 \mathrm{mag}$ in $v$ implies only a variation of the effective temperature of about $1 \%$. This is certainly much less than the uncertainty of the results from spectroscopic analysis obtained with currently available model atmospheres.

The result that Wolf-Rayet stars are basically stable is not a trivial one. Luminous Blue Variable stars are generally thought to be in a pre-Wolf-Rayet evolutionary stage. Obviously their stellar parameters are variable, yet their mass-loss rates-which could be regarded as a signature for instability - are similar to that of Wolf-Rayet stars.

\section{X-Ray Emission}

In principle, $\mathrm{X}$ rays are part of the stellar continuum energy distribution. However, there is not yet a quantitative theory that predicts $\mathrm{X}$ rays from single hot stars. Therefore, there is nothing to compare with the observations. What is known about X-ray emission from Wolf-Rayet stars has been summarized by Pollock (1987). However, in order to calculate a $\mathrm{X}$-ray luminosity for a given star he had to adopt a distance, a quantity which is often only poorly known. For a subset of stars with measured X-ray fluxes the bolometric corrections are known (Schmutz, Hamann, and Wessolowski 1989). For these stars we can calculate the distance independent-ratio $\mathrm{L}_{x} / \mathrm{L}_{b o l}$. In Figure 1 this ratio is given for 14 stars (excluding two non-detections). In several cases the original luminosity values given by the two references had to be adjusted for different assumptions of the stellar distance. From Figure 1 it is obvious that the $\mathrm{L}_{x} / \mathrm{L}_{b o l}$ ratio for most Wolf-Rayet stars is of the order of (a few) $10^{-7}$, 


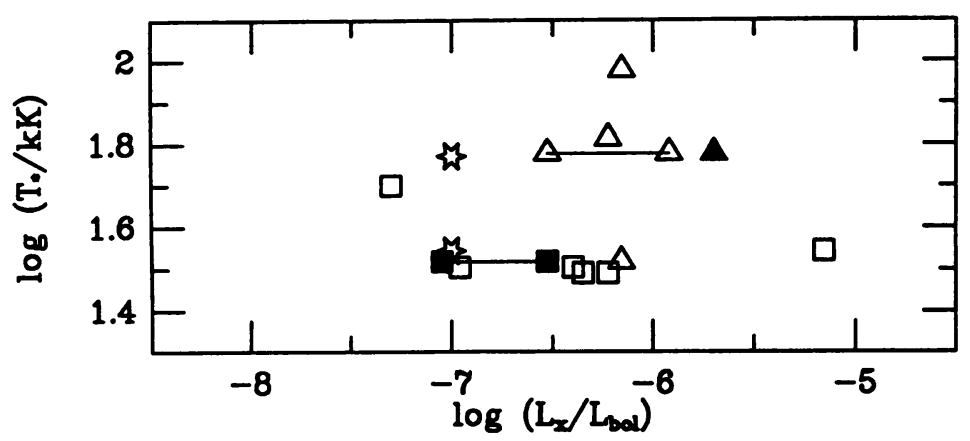

Figure 1: Ratio of X-ray luminosity (as given by Pollock 1987) to bolometric luminosity (as calculated by Schmutz, Hamann, and Wessolowski 1989 except for WR111 where $L_{b o d}$ is from Hillier 1989) for Wolf-Rayet stars common to both sources. Triangles denote WN E stars, squares WN L stars, and asterisks WC stars. Filled symbols denote binaries. The lines connect upper and lower limits of X-ray variables.

similar to that found for $O$ stars (Long and White 1980; Cassinelli et al. 1981). One of the strong sources $\left(L_{x} / L_{b o l}>10^{-6}\right)$ is a binary (V444 Cyg), and one is known to be variable and to have other peculiar properties as well (WR6). Only the extremely large X-ray flux from HD 93162 (WR25) is puzzling. For most of the Wolf-Rayet stars the conclusion of White and Long (1986), that the $\mathrm{L}_{x} / \mathrm{L}_{b o l}$ ratio for Wolf-Rayet stars is similar to that for OB stars, is confirmed.

\section{Continuum Colors}

The reason we are interested in knowing the intrinsic continuum energy distribution of a star is that the determination of the reddening due to interstellar extinction depends directly on it. For Wolf-Rayet stars it is not unrealistic to end up with an error of 0.15 mag in reddening if due care is not given to its evaluation (see below); this translates into an uncertainty of $50 \%$ in the luminosity determination. Even if this is not the largest source of uncertainty-usually the distance is-it is clear that one would like to avoid such a large error, if possible. There are several papers that have attempted to find a relation between spectral type and absolute magnitude and intrinsic colors for Wolf-Rayet stars (e.g. TorresDodgen and Massey 1988; Vacca and Torres-Dodgen 1990). These studies revealed some correlation but they also found a large scatter around the mean value for a given spectral type. The intrinsic scatter of the absolute magnitude-spectral type calibration seems to be as large as $1 \mathrm{mag}$. This implies an uncertainty of $60 \%$ if such a calibration is used for distance determinations. The intrinsic scatter of the $(b-v)_{0}$-spectral type calibration is of the order of 0.15 mag. Although we can add nothing to previous results regarding the absolute magnitudes, we are able to understand the origin of the scatter of the observed colors on the basis of theoretical models.

\subsection{CONTINUUM COLORS OF WC STARS}

Vacca and Torres-Dodgen (1990) found that the intrinsic $(b-v)_{0}$ colors of WC4 stars in the LMC span the range of 0.0 to $-0.5 \mathrm{mag}$. In view of this result it appears that the suggestion put forward by Smith, Shara, and Moffat (1990), that all WC4 stars are similar 


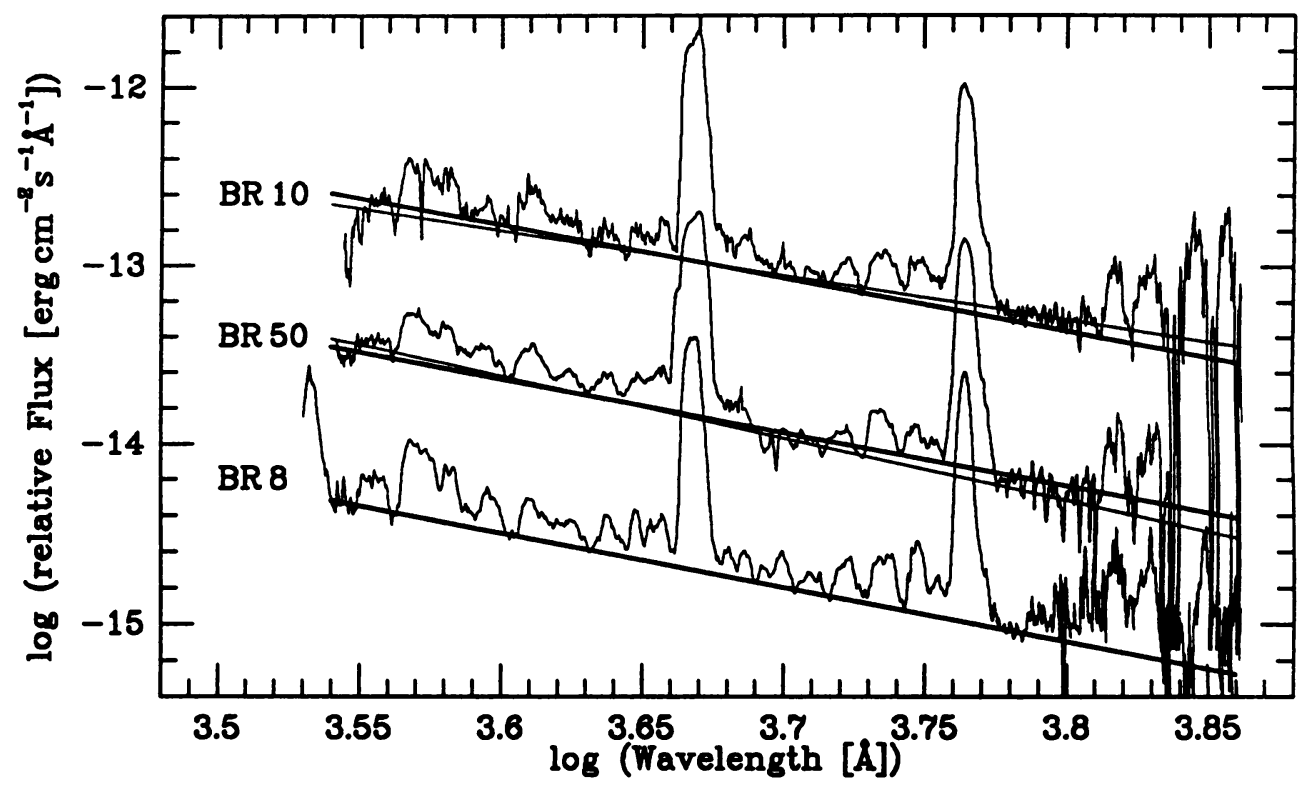

Figure 2: Dereddened relative fluxes of BR8, BR10, and BR50. The observations are from Torres and Massey (1987) and the $E_{B-V}$ values from Vacca and Torres-Dodgen (1990). The lines drawn indicate the estimated continua with slopes corresponding to $(b-v)_{0}=-0.2$ (thick lines) and -0.1 (thin line, BR10) and -0.3 (thin line, BR50).

and they all have an intrinsic color of $(b-v)_{0}=-0.3$, cannot be correct. However, because of the extreme blending of the numerous emission lines throughout the UV and the optical wavelength regions in WC stars-especially in WC4 stars which have the broadest line profiles-it is very difficult to define a continuum level. Thus, it could be that the large range in $(b-v)_{0}$ found by Vacca and Torres-Dodgen (1990) is due to incorrect determination of the continuum level. We can demonstrate that this is possible with the aid of results from atmosphere calculations. By examining the reddening-free indices $\Delta$ and $\eta$, as defined by Smith (1968) and Massey (1984), we have a means of estimating the errors in the continuum definition. From the work of Hillier (1989) and unpublished calculations we expect WC4 stars to have a power law continuum energy distribution in the UV/optical wavelength region with only small ( $\leqslant 0.03 \mathrm{mag}$ ) continuum discontinuities. Therefore, atmospheric models predict the line-free reddening-free indices to be close to 0.0 mag for all early type WC stars. This theoretical prediction is not confirmed by the observations. Massey (1984) found the line-free reddening-free indices of early WC stars to deviate by up to $0.3 \mathrm{mag}$ from 0.0 . We believe that these deviations are dominated by errors in defining the continuum level. The reddening-free indices are obtained from color differences, hence we may adopt an uncertainty of about $0.2 \mathrm{mag}$ in the $b-v$ colors. If we aiso allow for about 0.1 mag error in reddening (this relatively large error is adopted because reddening determinations from the $2200 \AA$ feature are also affected by the line blending problem) then uncertainties of up to $0.3 \mathrm{mag}$ in determining the intrinsic colors seem to be possible. Thus, the suggestion of Smith, Shara and Moffat (1990) that all LMC WC4 have similar colors cannot be refuted.

We may investigate their suggestion further by plotting in Figure 2 the dereddened fluxes of three WC4 stars, including the two with the most extreme $(b-v)_{0}$ colors, as determined 
by Vacca and Torres-Dodgen ( -0.0 and $-0.5 \mathrm{mag}$ ), and one for which an intermediate color ( $-0.2 \mathrm{mag}$ ) was determined. As mentioned above, according to the theoretical predictions WCE continua should follow power laws. Accordingly, in Figure 2 the continuum levels are approximated by straight lines in the log-log diagrams. The thick lines have slopes corresponding to $(b-v)_{0}=-0.2$. From this figure it is obvious that defining a continuum level for some WC stars is so difficult that the possibility that all three stars have the same intrinsic color of -0.2 cannot be excluded. However, a slope of -0.3 , as suggested by Smith, Shara, and Moffat (1990), is not consistent with the observed continuum of BR8 which has the best defined slope of the three stars shown in Figure 2. An intrinsic color of -0.2 would also agree with the results of the only published model for a WC star (Hillier 1989 and personal communication). The possibility of a similar slope for all three stars is strengthened by the intrinsic slope in the UV. For all LMC WC4 stars Vacca and Torres-Dodgen (1990) determined similar values of $\alpha$, where $\alpha$ is the slope in the UV $\log \lambda$ $\log F_{\lambda}$ diagram. Nevertheless, the stars may still be different. It might be that continua with slopes of -0.1 and -0.3 fit two of the stars in Figure 2 slightly better than -0.2. Additional evidence that the three stars are not identical is provided by their absolute magnitudes. The three stars BR8, BR10, and BR50 have $M_{v}=-3.4,-4.6$, and -4.1 mag, respectively. The differences in absolute magnitude are significant since the reddening corrections are not likely to be in error more than by $-0.1 \mathrm{mag}$, and therefore the absolute magnitudes are good within 0.3 mag.

To summarize, for LMC WC4 stars we cannot exclude the possibility that their intrinsic colors are similar. However, if their colors are identical then $(b-v)_{0}$ is more likely to be -0.2 rather than -0.3. There is evidence that there are differences between the WC4 stars-but this evidence is not conclusive enough to be considered a proof. For WC subtypes other than WC4 the same difficulty in defining a continuum level makes it unclear whether the range in for the $(b-v)_{0}$ values found by Vacca and Torres-Dodgen (1990) for each subtype is real or not.

\subsection{CONTINUUM COLORS OF WN STARS}

As in the case of WC stars, Vacca and Torres-Dodgen (1990) determined a large range of intrinsic $(b-v)_{0}$ colors within each WN spectral type. However, in contrast to the situation for WC stars, we are much more confident that it is possible to locate correctly the continuum levels in the spectra of WN stars. Therefore, there is no reason to question the reality of an intrinsic scatter in $(b-v)_{0}$ for each WN subtype. From the theoretical point of view this is a natural result since the predicted $(b-v)_{0}$, as well as equivalent widths of helium lines, is a function of two parameters, temperature and wind density (Schmutz 1988; see also Fig 3 of Hamann and Schmutz 1987), whereas the spectral type is a one-dimensional classification. Obviously, for WN stars there are different combinations of these two parameters realized in nature. Actually, it is more difficult to understand why it is apparently sufficient to use a one-dimensional description for WC stars.

If we trust the line-free colors measured from the absolutely calibrated spectra of WN stars by Massey (1984) and Torres-Dodgen and Massey (1988) then there is another observational fact to explain: Why do the reddening-free line-free indices of WN stars show a large range of values? Note that a range in $(b-v)_{0}$ colors, which we claim to be real, is not sufficient to explain this observation. If the continuum followed a power law then the reddening-free indices would still be close to zero. The answer comes naturally from the theoretical calculations. If the true opacity in an atmosphere is dominated by helium, as is the case for most WN stars, then for certain model parameter combinations the He II $n=4$ bound-free continuum introduces a considerable discontinuity at $3645 \AA$. The reddeningfree index $\Delta$ is essentially a measure of this continuum jump. Thus, the observed range 


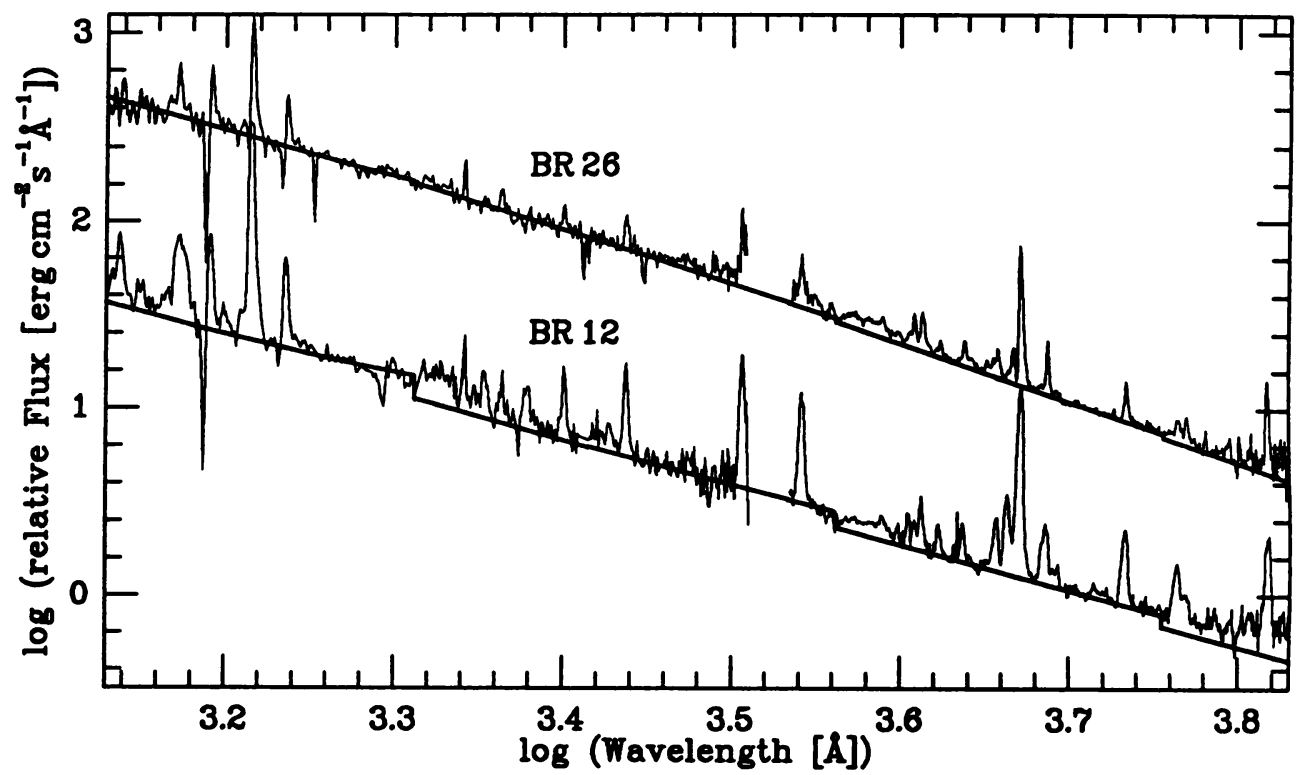

Figure 3: Dereddened relative fluxes of BR12 $\left(E_{B-V}=0.05\right.$ mag $)$ and BR26 $\left(E_{B-V}=0.04\right.$ mag $)$ compared with theoretical flux distributions predicted for these stars. The optical observations are from Torres-Dodgen and Massey (1988) and the combined IUE spectra from Vacca and TorresDodgen (1990).

of values of this parameter implies that there are WN stars with no significant continuum jumps and there are other WN stars with discontinuities of up to 0.3 mag in emission.

Atmospheric models predict a close correlation between $(b-v)_{0}$ and $D_{3645}$, the continuum discontinuity at $3645 \AA$. If the model calculations yield a flat continuum (small $\left.(b-v)_{0}\right)$ then the jump at $3645 \AA$ is found to be in emission. This correlation also implies that the range in $(u-b)_{0}$ is much smaller than that in $(b-v)_{0}$ :

$$
\text { WN stars: } \begin{aligned}
& (u-b)_{0}=-0.22 \ldots-0.27 \mathrm{mag} \\
& (b-v)_{0}=-0.05 \ldots-0.35 \mathrm{mag}
\end{aligned}
$$

The small range in $(u-b)_{0}$ and the relation between $(b-v)_{0}$ and $D_{3645}$ allow us to formulate an expression to calculate the interstellar reddening from observed $u-b$ and $b-v$ colors alone (Vacca and Schmutz, this symposium).

\section{Continuum Fits}

In section 4 we used theoretical continuum energy distributions to predict intrinsic colors, but we have not yet shown that the predictions actually fit the observations. Two examples of observed and theoretical continuum energy distributions are shown in Figure 3. The examples shown are among the best at hand. Other comparisons are usually of similar quality, except that for some either the UV or the optical observations are not available, or in other cases the UV and optical fluxes do not agree very well. In some cases the 


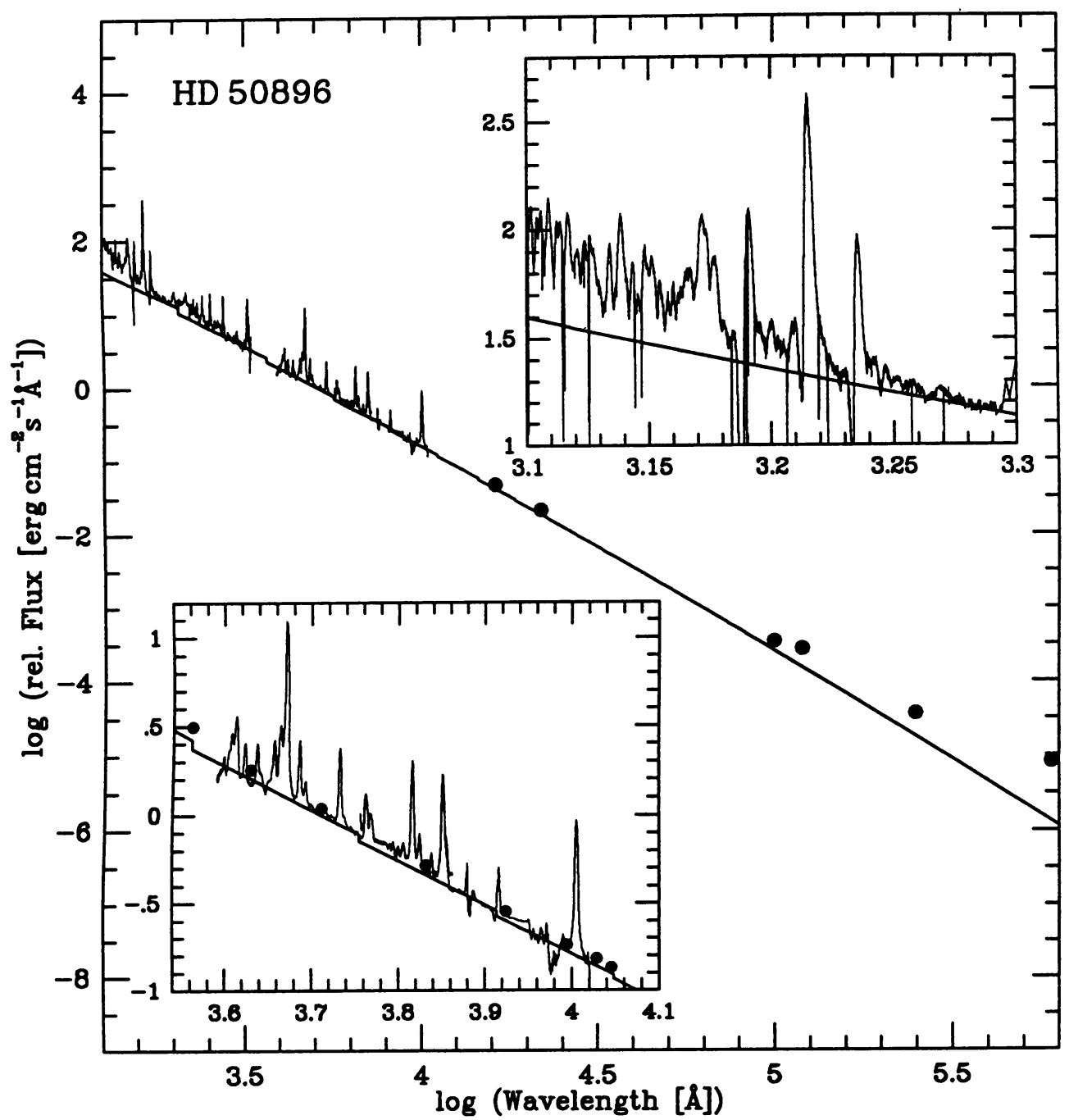

Figure 4: Observed spectrum of HD 50896 (WR6) dereddened by 0.07 mag. The UV spectrum is a low-resolution $I U E$ observation. The $H$ and $K$ photometric values are from Allen, Swings, and Harvey (1972), and the $10 \mu \mathrm{m}$ flux is obtained from Barlow, Smith, and Willis (1981). The IRAS observations at 12,25 , and $60 \mu \mathrm{m}$ (van der Hucht et al. 1985) do not agree with either an extrapolation of the observations or with the predicted spectrum. Probably the flux measured through the large aperture of IRAS is contaminated by (dust?) emission from the ring nebula S308. Inset top: Enlargement of the IUE-SWP wavelength region. The high-resolution spectrum published by Howarth and Phillips (1986) is the average from 52 IUE spectra. Inset bottom: Enlargement of the optical/near-IR wavelength region. The optical spectrum is an observation by the author and the near-IR observation is taken from Vreux, Dennefeld, and Andrillat (1983). The dots mark photometric ubv fluxes as measured by Smith (1968) and near-IR values from Cohen, Barlow, and Kuhi (1975). 
models fit most of the observed wavelength range but deviate over small spectral regions (e.g., around $2200 \AA$ ). In Figure 3 we show fits to two LMC WN stars. Both stars are only slightly reddened and therefore the adopted reddening law has practically no influence on the observed continuum energy distribution. The stellar parameters for the models of the two stars are from Koesterke et al. (1990) and the corresponding continuum energy distributions have been taken from the flux-grid published by Schmutz et al. (1990). The fits shown illustrate one continuum in which there are essentially no discontinuities and one that exhibits strong emission jumps. Note that if there is a discontinuity at 3645 $\AA$ there are also discontinuities expected at all other observable He II edges. The models predict a correlation not only between $(b-v)_{0}$ and $D_{3645}$, but also between these quantities and the strengths of the HeII lines. Therefore, if the discontinuity is strongly in emission the emission lines of the corresponding series will be strong, will merge longward of the continuum edges, and will form a pseudo-continuum. This will give the impression that the discontinuity is at a longer wavelength than theoretically expected.

The predicted continua are clearly excellent fits to the observations with the exception of deviations at each end of the displayed wavelength region. The disagreement in the UV will be discussed below; the discrepancy in the red wavelength range is due to the deteriorating quality of the observation. The latter can be demonstrated to be true because the theoretical spectrum fits additional observations at even longer wavelengths ( $J H K$ fluxes).

For the lightly reddened Galactic star HD 50896 (WR6) a comparison over a large wavelength range-3.7 dex in wavelength-is shown in Figure 4. For this comparison of observation and theory a model continuum of Hillier (personal communication; see also Hillier $1987 b$ ) was used. This figure demonstrates again how well theory fits the observation. From such fits the reddening due to interstellar extinction can be determined to an accuracy of $0.01 \mathrm{mag}$, provided the UV extinction law is known and there are no systematic errors in the predicted spectrum. As an illustration that theoretical spectra also fit the red wavelength region, and in particular the discontinuity at $5695 \AA$, the lower inset in Figure 4 gives an enlargement of the optical/near-IR wavelength region. The theoretical spectrum shown in this figure is taken from the work of Hamann, Schmutz, and Wessolowski (1988).

\section{UV Pseudo-Continuum Formed by Fe Lines}

For all stars that display strong emission lines the theoretical continua do not fit the observations for wavelengths shorter than about $1500 \AA$, the predicted continua being always too low. Examples of this inconsistency can be seen in Figures 3 and 4. This is not a result of insufficient resolution of the UV observations. The upper inset of Figure 4 shows that the discrepancy remains even if high-resolution observations are used for the comparison. The reason for the disagreement has been found independently by two groups (Koenigsberger and Auer 1985; Nugis and Sapar 1985): In the wavelength region shortward of 1500 $\AA$ numerous $\mathrm{FeV}$ and $\mathrm{FeVI}$ lines are present and form a pseudo-continuum. In Figure 5 a comparison is shown between the normalized spectrum of HD 50896 and the combined $\mathrm{Fe}$ / Fe vi laboratory spectrum (Ekberg $1975 a, b$ ). The basic shape of the emission spectrum is very similar to that of the iron intensity distribution. This figure demonstrates that it is quite plausible that iron emission is the source of the pseudo-continuum. Most of the remaining (strong) emission lines have well-known identifications (Willis et al. 1986).

\section{Comparison Between Predicted and Observed Helium Line Strength Ratios}

The model that fits a given observation is determined by the strength of one HeI and one 


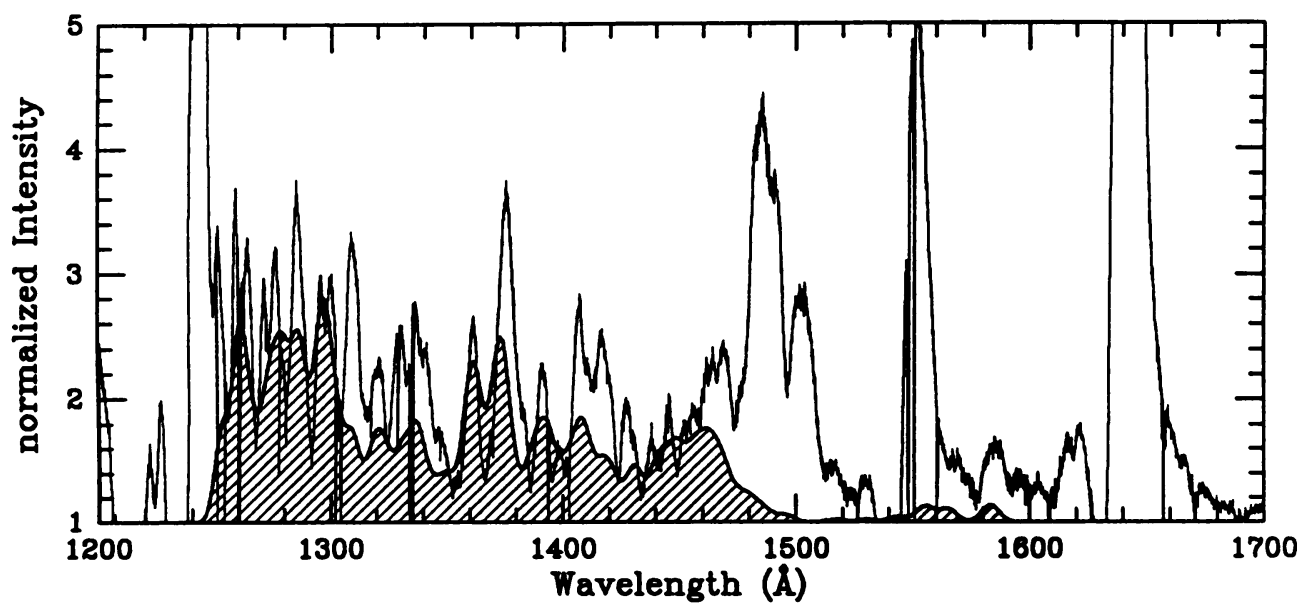

Figure 5: Pseudo-continuum in the spectrum of HD 50896. The plotted spectrum is the observed dereddened flux divided by the predicted continuum energy distribution (see Fig. 4). The shaded area is the emission spectrum of $\mathrm{FeV}$ and $\mathrm{FeVI}$ as observed in the laboratory. The $\mathrm{Fe} / \mathrm{FeVI}$ spectrum broadened to $1000 \mathrm{~km} \mathrm{~s}^{-1}$ was obtained from Koenigsberger (1988).

He II line, e.g. He I $\lambda 5876$ and He II $\lambda 4686$ (see Schmutz, Hamann, and Wessolowski 1989). However, there are usually more He I and He II lines observed than just the minimum of two lines needed for the analysis. Conti and Massey (1989) demonstrated that there is a well defined correlation between the observed line strengths of He II transitions. It is possible therefore, to test the reliability of the models by comparing the observed and predicted correlations.

In Figure $6 a$ the theoretical and observed correlations between the equivalent widths of He II $\lambda 1640$ and He II $\lambda 4686$ are compared. The theoretical correlation is tighter than the observed one. This can be understood as the result of errors in measuring the equivalent widths. However, the observed values appear to differ systematically from the predicted ones for Galactic WN E stars with strong lines. Presumably, the iron pseudo-continuum is stronger in the Galactic stars than in the LMC stars due to the higher metal abundance in the Galaxy and, therefore, the HeII $\lambda 1640$ equivalent widths in Galactic stars may be underestimated.

In Figure $6 b$ the theoretical and observed correlations between He II $\lambda 5411$ and He II $\lambda 4686$ are compared. Both correlations are about equally well defined. This indicates that the observational scatter in Figure $6 a$ is predominantly due to errors in the He II $\lambda 1640$ equivalent width measurements. In Figure $6 b$ a slight discrepency between the observation and theory is perceptible. An example of the disagreement between the observed and predicted line ratios is found in the case of HD 50896. The profile fits published by Hamann, Schmutz, and Wessolowski (1988) show good agreement for the He II $\lambda \lambda 1640,4686$ lines, a result which implies that the ratio between these two lines is predicted correctly. However the theoretical profile for HeII $\lambda 5411$ is too narrow, and thus, the theoretical equivalent width is too small. A plausible explanation for the disagreement would be a contribution to the He II $\lambda 5411$ equivalent width by another line. However, there is no good candidate for such a line. Although Hillier (1987b) fits the observed width of He II $\lambda 5411$, his predicted He II line ratio is incorrect as well. He calculates a He II $\lambda 1640$ line which is too broad, and thus finds an equivalent width which is too large. 

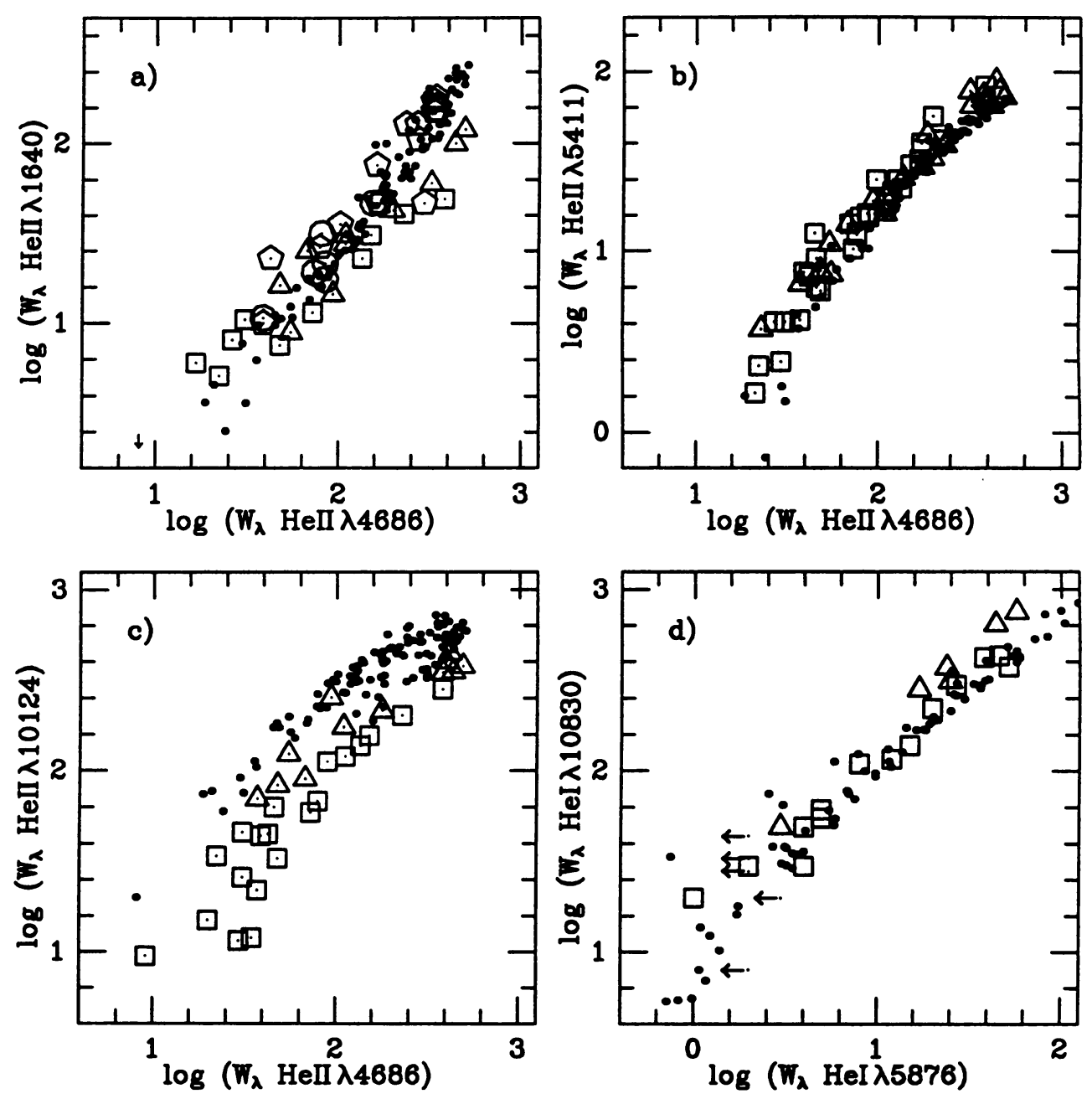

Figure 6: Comparison between observed and theoretical correlations of helium line equivalent widths; a) upper left panel: He II $\lambda 1640$ versus He II $\lambda 4686$; b) upper right panel: He II $\lambda 5411$ versus He II $\lambda 4686$; $c$ ) lower left panel: He II $\lambda 10124$ versus He II $\lambda 4686$; d) lower right panel: He I $\lambda 10830$ versus He I $\lambda 5876$. The theoretical equivalent widths are marked with small filled dots. The observed values are marked with open symbols: squares-Galactic WN L stars; circles-LMC WN L stars; triangles-Galactic WNE stars; pentagons-LMC WNE stars. The arrow in panel (a) marks a value where the theoretical equivalent width of HeII $\lambda 1640$ is in absorption; the arrows in panel (d) mark upper limits for the observed equivalent widths of He I $\lambda 5876$. The references for the observed equivalent widths are: Smith and Willis (1983); Conti and Massey (1989); Conti and Morris (1990); Vreux, Dennefeld, and Andrillat (1983); Vreux et al. (1989); Conti, Massey, and Vreux (1990); Vreux, Andrillat, and Biemont (1990). 

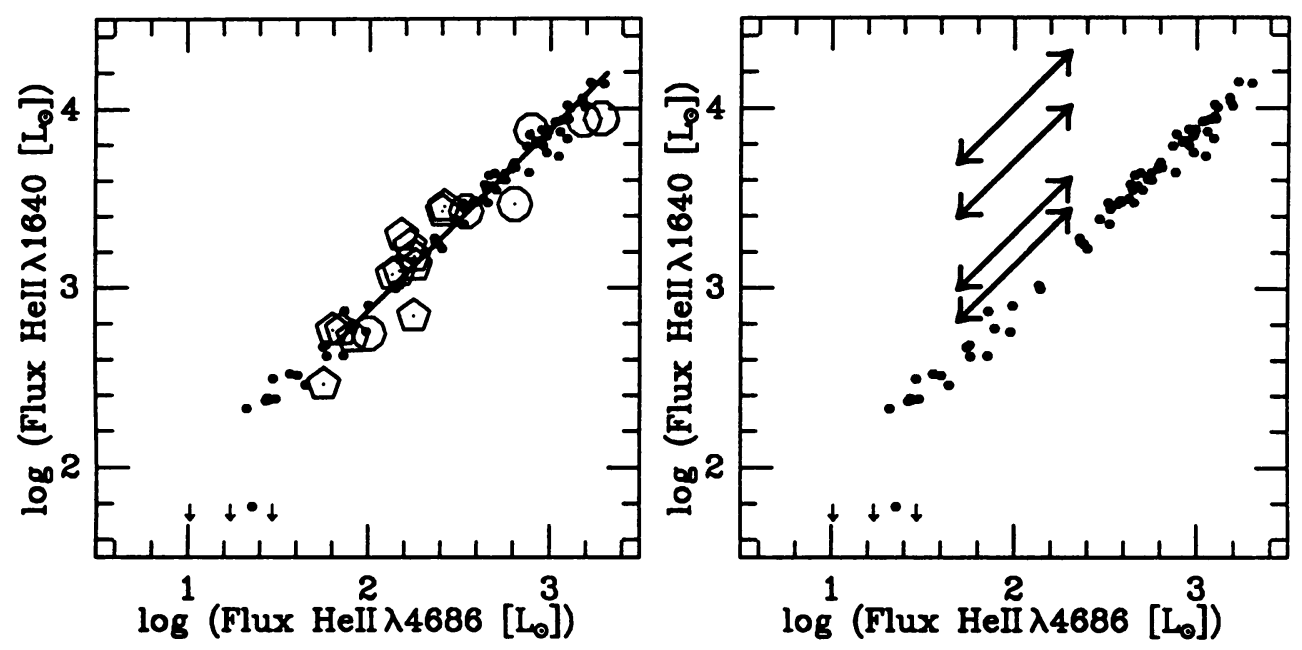

Figure 7: Correlation of He II $\lambda 1640$ and He II $\lambda 4686$ line fluxes: a) Comparison of observed and theoretical correlations; b) Comparison between the correlation predicted by the Kiel models (dots) with the flux ratios predicted by Bhatia and Underhill (1986) (arrows).

In Figure $6 c$ the theoretical and observed correlations between He II $\lambda 10124$ and He II $\lambda 4686$ are compared. It is obvious that the two correlations do not agree. Since He II $\lambda 4686$ is involved in the two comparisons where agreement is found, the problem must lie with the HeII $\lambda 10124$ line. The comparisons in Figures $6 a$ and $b$ demonstrated that the populations of HeII $n=2,3,4$, and 7 are calculated correctly in the models. The HeII $\lambda 10124$ transition is between levels $n=4$ and 5 . Theoretically, it is difficult to understand how the model population of He II $n=5$ could be wrong while other He II level-populations are correct. Yet, there is no obvious reason why the observations should be in error. To make the disagreement even more mysterious, the detailed analysis of HD 50896 by Hamann, Schmutz, and Wessolowski (1988) yields the correct intensity for this line. The entry in Figure $6 c$ corresponding to this star lies in the upper right corner at about the only place where observational and theoretical values overlap.

In the case of HeI lines data are available only for HeI $\lambda 10830$ and $\mathrm{HeI} \lambda 5876$. The comparison between the theoretical and observed correlations of these lines is shown in Figure $6 d$. Good agreement is found except for strong-line stars where theory predicts $\mathrm{He}$ I $\lambda 5876$ too strong. The reason for this discrepency can be traced to the fact that the blending of HeI $\lambda 5876$ with the lines of the He II n-5 series, which form a pseudo-continuum at the wavelength of the HeI line (see lower inset Fig. 4), is not taken into account in the model calculations. Calculations simulating the effect of the blends by artificially including the He II $n=5$ continuum at the wavelength of the He I line yield a smaller He I $\lambda 5876$ equivalent width. The theoretical He I line ratio then agrees with the observed one for WN stars with strong lines as well.

Conti and Morris (1990) also measured line fluxes. In Figure 7a the correlation between the observed dereddened line fluxes is compared with the theoretical correlation. The solid line is the mean correlation adopted by Conti and Morris (1990) and corresponds to an average line flux ratio of about 7.6. However, theory predicts a range of line flux ratios. This range translates into an intrinsic uncertainty of $0.07 \mathrm{mag}$ in $E_{B-V}$ if the average line 
flux ratio is used to derive the color excess due to interstellar reddening.

In Figure $7 b$ the theoretical line flux correlation between He II $\lambda 1640$ and He II $\lambda 4686$ is compared with some of the line-flux ratios predicted by the models of Bhatia and Underhill (1986); the smallest ratio predicted is $(F(1640) / F(4686)=13.4)$. Obviously, Bhatia and Underhill (1986) predict ratios different from those calculated with the model atmospheres used elsewhere in this paper. The reason for the difference is that, given the physical conditions assumed by Bhatia and Underhill (1986) in the line emitting region, collisional rates play an important role in the equations for statistical equilibrium of the level populations. This contrasts with the inherent assumptions of the Kiel/JUA models, in which the rate equations are dominated by radiative processes. Since neither the large ratios nor the wide range of $\mathrm{He}$ II line flux ratios calculated by Bhatia and Underhill (1986) agree with the observations we conclude that their model does not describe correctly the physical conditions in Wolf-Rayet atmospheres.

\section{Conclusion}

In Section 5 it was found that the predicted continuum energy distributions fit the observations and in Section 7 it was demonstrated that the observed correlations between several helium lines are reproduced well by the "standard" model calculations. This agreement provides strong evidence for the reliability of the model calculations. Therefore, we may use the model results to judge the accuracy of different methods of determining reddening. We conclude by presenting a summary of the various methods, their intrinsic errors, and the expected uncertainty in their application to actual observations (Table 1).

Table 1. Reddening determinations for WN stars.

\begin{tabular}{|c|c|c|}
\hline method & $\begin{array}{c}\text { intrinsic uncertainty } \\
\text { (not Gaussian) }\end{array}$ & $\begin{array}{l}\sigma \text { in practice } \\
\text { (Gaussian) }\end{array}$ \\
\hline $\begin{array}{l}E_{B-V} \text { from fit to model } \\
\text { continuum }\end{array}$ & 0. & $\begin{array}{c} \pm 0.02 \\
\text { but: UV reddening law }\end{array}$ \\
\hline $\begin{array}{l}E_{B-V}=\mathcal{F}(u-b, b-v) \\
\text { (Vacca and Schmutz, this sym- } \\
\text { posium) }\end{array}$ & \pm 0.02 & $\begin{array}{l} \pm 0.07 \\
\text { but: only for helium dominated } \\
\text { atmospheres and } T_{*} \geq 35 \mathrm{kK}\end{array}$ \\
\hline $\begin{array}{l}E_{B-V} \text { from } 2200 \AA \text { feature } \\
\text { (e.g. Vacca and Torres-Dodgen 1990) }\end{array}$ & 0. & $\begin{array}{ll} & \pm 0.05 \ldots 0.1 \\
\text { but: } & \text { UV reddening law }\end{array}$ \\
\hline $\begin{array}{l}E_{B-V} \text { from } \frac{F(1640)}{F(4686)} \\
\text { (Conti and Morris 1990) }\end{array}$ & \pm 0.07 & $\begin{array}{c} \pm 0.1 \\
\text { but: UV reddening law }\end{array}$ \\
\hline$E_{B-V}$ from $<(b-v)_{0}>_{\text {Sp. }}$. & \pm 0.15 & \pm 0.15 \\
\hline
\end{tabular}

Acknowledgements. I thank my colleagues Drs. John Hillier, Pete Storey, and Bill Vacca for many discussions and valuable suggestions. Thanks also go to Bill Vacca and Lorraine Volsky for editorial assistance. I acknowledge Gloria Koenigsberger for providing the Fe $\mathrm{V}$ and Fe VI data, Ian Howarth for sending the IUE high-resolution spectrum of HD 50896, and the Astronomical Data Center at the Goddard Space Flight Center for making available the spectra published by Torres and Massey (1987) and Torres-Dodgen and Massey (1988). This work was supported by the Swiss National Foundation and by the National Science Foundation (U.S.) through Grant AST88-02937. 


\section{References}

Allen, D. A., Swings, J. P., and Harvey, P. M. 1972, Astr. Ap., 20, 333.

Barlow, M. J., Smith, L. J., and Willis, A. J. 1981, M.N.R.A.S., 196, 101.

Bhatia, A. K., and Underhill, A. B. 1986, Ap. J. Suppl., 60, 323.

Cassinelli, J. P., et al. 1981, Ap. J., 250, 677.

Conti, P. S., and Massey, P. 1989, Ap. J., 337, 251.

Conti, P. S., Massey, P., and Vreux, J. M. 1990, Ap. J., 354, 359.

Conti, P. S., and Morris, P. W. 1990, A. J., 99, 898.

Cohen, M., Barlow, M. J., and Kuhi, L. V. 1975, Astr. Ap., 40, 291.

Ekberg, O. 1975a, Phys. Scripta, 11, 23.

- 1975b, Phys. Scripta, 12, 42.

Hamann, W.-R., and Schmutz, W. 1987, Astr. Ap., 174, 173.

Hamann, W.-R., Schmutz, W., and Wessolowski, U. 1988, Astr. Ap., 194, 190.

Hillier, D. J. 1987a, Ap. J. Suppl., 63, 947.

—. 1987b, Ap. J. Suppl., 63, 965.

—. 1989, Ap. J., 347, 392.

Howarth, I. D., and Phillips, A. P. 1986, M.N.R.A.S., $222,809$.

Koenigsberger, G. 1988 Rev. Mex. Astr. Astrof., 16, 75.

Koenigsberger, G., and Auer, L. H. 1985 Ap. J., 297, 255.

Koesterke, L., Hamann, W.-R., Schmutz, W., and Wessolowski, U. 1990, in preparation.

Long, K. S., and White, R. L. 1980, Ap. J. (Letters), 239, L65.

Massey, P. 1984, Ap. J., 281, 789.

McCandliss, S. R. 1988, Ph. D. Thesis, University of Colorado.

Monderen, P., De Loore, C. W. H., van der Hucht, K. A., and van Genderen, A. M. 1988, Astr. Ap., 195, 179.

Nugis, T., and Sapar, A. 1985, Sov. Astr. Let., 11, 188.

Pollock, A. M. T. 1987, Ap. J., 320, 283.

Schmutz, W. 1988, in Lecture Notes in Physics Vol. 305, IAU Colloquium 108, p. 133.

Schmutz, W., Hamann, W.-R., and Wessolowski, U. 1989, Astr. Ap., 210, 236.

Schmutz, W., and Vacca, W. D. 1990, Astr. Ap. Suppl., in preparation.

Schmutz, W., Vogel, M., Hamann, W.-R., and Wessolowski, U. 1990, Astr. Ap., in preparation (model fluxes are available from the author).

Smith, L. J., and Willis, A. J. 1983, Astr. Ap. Suppl., 54, 229.

Smith, L. F. 1968, M.N.R.A.S., 140, 409.

Smith, L. F., Shara, M. M., and Moffat, A. F. J. 1990, Ap. J., 348, 471.

St-Louis, N., et al. 1989, Astr. Ap., 226, 249.

Torres, A. V., and Massey, P. 1987, Ap. J. Suppl., 65, 459.

Torres-Dodgen, A. V., and Massey, P. 1988, A. J., 96, 1076.

Vacca, W. D., and Torres-Dodgen, A. V. 1990, Ap. J. Suppl., 73, in press.

van der Hucht, K. A., et al. 1985, in Birth and Evolution of Massive Stars and Stellar Groups, ed. W. Boland and H. van Woerden (Dordrecht: Reidel), p. 167.

van Genderen, A. M., van der Hucht, K. A., and Larsen, I. 1990, Astr. Ap., 229, 123.

Vreux, J. M., Andrillat, Y., and Biemont, E. 1990, Astr. Ap., preprint.

Vreux, J. M., Dennefeld, M., and Andrillat, Y. 1983, Astr. Ap. Suppl., 54, 437.

Vreux, J. M., Dennefeld, M., Andrillat, Y., and Rochowicz, K. 1989, Astr. Ap. Suppl., 81, 353.

Westerlund, B. E. 1966, Ap. J., 145, 724.

White, R. L., and Long, K. S. 1986, Ap. J., 310, 832.

Willis, A. J., van der Hucht, K. A., Conti, P. S., and Garmany, C. D. 1986, Astr. Ap. Suppl., 63, 417. 


\section{DISCUSSION}

Koenigsberger: Do you have any means of relating the physical position within the wind of the gas producing the spectrum you model with the radius of the underlying stellar core? Schmutz: Yes, we do, most of John Hillier's papers include graphs that show the radial distribution of the line emissions.

Underhill: It is strange that your calculations indicate that collisional transitions may be neglected in your model atmospheres because most of your line emission comes from volume elements where the density is greater than $10^{9} \mathrm{~cm}^{-3}$. From debugging the Bhatia and Underhill programs I know that the collisional rates are comparable to the radiative rates for many transitions when $N_{e}>10^{9} \mathrm{~cm}^{-3}$ in our one-point models. You should always carry all terms in the equations of statistical equilibrium and let the computer find which are negligible. Bhatia and Underhill included 5 different kinds of transition. This is the most complete study of statistical equilibrium made for modelling WR atmospheres.

$S c h m u t z$ : I did not say collisions can be neglected. Collisional rates are of course included in our model calculations and they play a role to recover LTE at large optical depth. However, in the line-formation regions it turns out that for the physical conditions we are modelling the formation of the $\mathrm{HeII}$ lines is dominated by recombination and photoionization. The special physical conditions you assume are needed in order that collisions become important. This is exactly the reason why you and we predict different ratios for the $\mathrm{HeII}$ lines. Thus, we have a tool to discriminate between the models. I am afraid this test is not in favour of the ideas you are putting forward.

Owocki: (1) Your "nonvariability" refers only to overall luminosity at very long time scales. There are numerous spectral observations indicating few $\%$ variability on dynamical $(\sim 1$ day) time scales. (2) X-rays are normally thought to arise from variable wind situations (i.e. shocks). What do you mean by "normal" X-ray?

Schmutz: (1) I am well aware that there are real variations in profiles and magnitude. The point that I wanted to make is that most vary only so little that it does not affect the results of spectroscopic analyses. (2) By "normal" I mean the X-ray level of "most" of the stars.

Schulte-Ladbeck: I have a question about the continuum energy distribution. Some WR stars may have flattened atmospheres as evidenced by polarimetry (maybe due to rotation)! Your models, I assume, use spherical symmetry. Do you have any idea how the continua would change for a flattened model?

Schmutz: Our models do assume spherical symmetry and we do not (yet) know what the influences of a flattened atmosphere would be. However, for some stars polarimetric measurements indicate basically zero polarisation, implying that at least some WR atmospheres are spherically symmetric, i.e. our models are valid for these stars.

Pollock: I would advise you against taking too literally the normality of the X-ray emission from WR stars because most of the single WR stars, those that are not binaries or nonthermal radio sources, are pretty weak in X-rays and e.g. the point for WR136 (that was about the only one from a single star that appeared on your graph) is in fact a non-detection and I would not put it there if I were you.

Schmutz: I just wanted to say, there is a belief that WR stars are not normal, i.e. below the limit of normal $O$ stars, and actually they are not. Most of them are in the $L_{x} / L_{b o l}=10^{-7}$ region, with large scatter. 